\title{
Fetal Echocardiography as a Screening Method for Detection of Congenital Heart Disease
}

\author{
Hesham El Sheikh, Ahemd E. Shalaan, Esraa A. Abdelazeem
}

Department of Radiology, faculty of medicine Benha University, Egypt

Correspondence to: Esraa A. Abdelazeem, Department of Radiology, faculty of medicine Benha University Egypt.

Email:

dr_esraa2377@yahoo.com

Received: 22 August 2019

Accepted: 27 October 2019

\begin{abstract}
:
Background: Congenital heart diseases (CHDs) are among the most common congenital abnormalities. They are frequently missed on routine obstetric ultrasound (US) examination. Although fetal echocardiography is reserved only for high-risk cases, many cases with cardiac abnormalities are born to mothers with no identifiable risk factors. Aim of work: is to assess fetal echocardiography as a screening method for prenatal detection of cardiac abnormalities in an un-selected group of pregnant females. Patients and Methods: During the period of March 2108 to March 2019, 150 fetuses for 149 un-selected pregnant female (there was a set of twin pregnancy) underwent a detailed fetal echocardiographic examination; cases that had maternal, fetal or familial risk factors for CHD were categorized as high risk, others with no known risk factors were low risk. Results: Structural heart defects were found in $6(4 \%)$ fetuses in both high and low risk groups. The prevalence of prenatally detected cardiac abnormalities was $3.5 \%$ in the low-risk group and $5.5 \% \%$ in the high-risk group. The difference in the incidence of CHD between the two groups was not statistically significant $(\mathrm{P}=0.4)$. Conclusion: Our study shows no difference in the incidence of CHDs between pregnancies associated with high risk factors compared to those with no risk factors, so we advocate fetal echocardiography to be included
\end{abstract} in any fetal anomaly scanning for every pregnant woman regardless the presence of risk factors. Keywords: Congenital heart diseases (CHDs), fetal echocardiography, prenatal screening. 

Introduction

Congenital heart diseases (CHDs) are among the most common congenital abnormalities, affecting approximately 6-9 per 1000 newborns, and are a leading cause of infant morbidity and mortality. ${ }^{(1)}$

CHD is defined as a gross structural abnormality of the heart or intra-thoracic great vessels that is actually or potentially of a functional significance. ${ }^{(2)}$ This definition excludes abnormalities that do not impact cardiac function, arrhythmias, and conditions not always present at birth such as hypertrophic or dilated cardiomyopathies. ${ }^{(3)}$

CHDs are responsible for about $40 \%$ of perinatal deaths of which more than $20 \%$ of deaths occur in the first month of life. CHDs therefore, significantly contribute to the economic burden on health care systems. Prenatal fetal echocardiography is the only means of detecting such defects. $12^{(4)}$

Certain groups of pregnancies are at increased risk for $\mathrm{CHD}$ and these patients should be referred for a focused echocardiogram. ${ }^{(5)}$

Although it is the high-risk groups that are referred for a fetal cardiology opinion, most cases of congenital heart disease will occur in a low-risk population ${ }^{(6)}$. The only hope for these patients to be detected is for something abnormal being recognized during the obstetric scan. For this reason, the concept of "screening" the fetal heart in a simplified fashion during routine obstetric scanning was introduced. ${ }^{(7)}$

The concept of prenatal screening for CHD was introduced in the UK in $1986^{\text {(8) }}$ following the results of a French study ${ }^{(9)}$. Subsequently, a concentrated screening program was set up in 10 obstetric centers in the South East Thames Region in 1988. ${ }^{(10)}$

Early detection of CHDs in prenatal period by fetal echocardiography is of paramount importance in management of such cases. Prenatal diagnosis helps family in making a decision regarding the outcome of pregnancy including termination.

It also decreases mortality and morbidity by smooth transition between pre and post-natal states by providing the appropriate medical care immediately after birth. ${ }^{(4)}$

\section{Aim of work}

Our objective is to assess fetal echocardiography as a screening method for prenatal detection of CHD. 


\section{Patients and methods}

This was a prospective study; the data was collected during the period of March 2018 to March 2019. The research ethical committee of Benha faculty of medicine approved the protocol of this study.

\section{Study population}

Screening for CHDs was offered to all pregnant women routinely attending the obstetric ultra-sonographic unit in the radiology department at Benha University Hospital. Of 152 pregnant women underwent fetal echocardiography, only 149 who met the inclusion criteria; three cases were excluded due to intra-uterine fetal death (IUFD).

Of the 149 pregnant women that were included, there was a set of a twin pregnancy, so our study population included 150 fetuses.

The cases were then categorized as high and low risk; the high-risk group were selected in accordance with the previously identified selection criteria that placed the mothers at risk for carrying fetuses with heart disease as described by Donofrio et al. ${ }^{(11),}$ low-risk group included pregnant women with no known risk factors for CHD.

\section{Fetal echocardiographic examination}

○ All studies were done trans-abdominally with a 3.5 to $5 \mathrm{MHz}$ curved array transducer of (General electric company (GE) health care model GE Logic P6, and p7 Pro, USA).

$\circ$ Before examination of the fetal heart, routine obstetric US was performed for assessment of gestational age and other fetal anomalies.

○ Fetal viability was determined by either M-mode or direct visualization of the fetal cardiac activity.

$\circ$ We considered the method adopted by Eggeb $\varnothing$ and his colleague in assessing the fetal laterality within the uterus, this method depends only on two variables; fetal presentation and rotation of the interventricular septum related to a line through the fetal chest. ${ }^{(12)}$

$\circ$ The presentation of the fetus within the uterus was assessed by obtaining longitudinal image of the cervix and uterine fundus. Following this, we got a fourchamber view of the heart by obtaining a transverse view of the fetal thorax; a line drawn through the inter-ventricular septum forms an angle with an imaginary line from the spine to the sternum. The interventricular line is either orientated clockwise or counter-clockwise related to the line through the chest.

o Once we have determined the laterality of the fetal body, we obtained a transverse 
view of the fetal upper abdomen and checked the arrangement of vessel and abdominal viscera thus assessing visceral situs.

- We ascertained if the stomach and heart are in their correct respective locations, i.e., the stomach is on the left site and the cardiac axis points to the left.

o We examined the fetal heart in various imaging planes with continuous sweeping of the transducer. In this technique, we followed the guidelines recommended and updated by ISUOG for cardiac screening in mid-gestation; "basic" (four-chamber view) and "extended basic" (views of the outflow tracts) scan ${ }^{(13)}$ and routinely obtained the following five transverse planes views: transverse view of the upper abdomen, the four-chamber view, the left ventricular outflow tract view, the right ventricular outflow tract view and the three-vessel view.

○ We performed color Doppler that aided in visualization of normal anatomy and detection of small vessels such as pulmonary veins entering the left atrium. It also added information concerning detection of blood flow, direction of flow (antegrade, retrograde or bidirectional).

○ Starting with a good 2D image was essential for obtaining a high color-Doppler image quality that was also affected by the size of color box, the velocity range and color gain. Initially, low color gain and high pulse repetition frequency (PRF) were used and continuously adjusted as needed.

- We reviewed the screening sonograms, and then we analyzed the abnormalities seen in various screening views.

o Fetal echocardiography findings and diagnosis were correlated with post-natal diagnosis based on clinical examination and echo-cardiography study.

\section{Results}

This study included 150 cases of un-selected pregnant females; their ages ranged from 21 to 38 years with the mean age was 28 years. The cases were categorized as high and low risk subgroups. 36 out of 150 women (24\%) were categorized as high risk; the remaining 114 women (76\%) were low risk (Fig.1)

\section{Risk Factors}

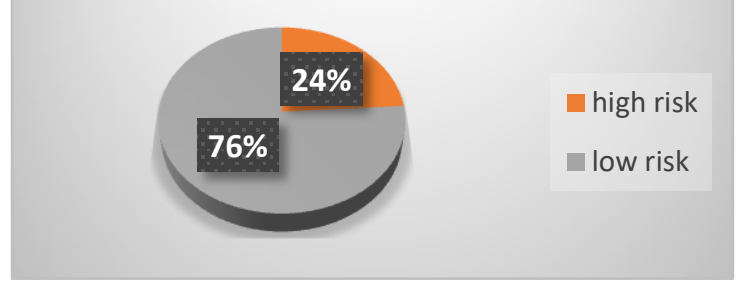

Fig. 1: The percentage of high- and low-risk subgroups among the study population.

The most commonly encountered risk factor was positive consanguinity constituting $10 \%$ of the study population, followed by 
Benha medical journal vol. 38, special issue (Radiology), 2021

maternal old age (>35 years old) constituting 6\%. Pregnant females with coexisting maternal disease (diabetes mellitus or maternal infection) recorded $4 \%$ of the study population, $1.3 \%$ had a previous baby with CHD. Assisted conception was reported in $2 \%$ of the study population and there was a set of monochorionic twin $(0.6 \%)$ of the study population (Table 1).

Of the 150 fetuses underwent echocardiographic examination, 6 cases $(4 \%$ of the study population) were found to have cardiac abnormalities ( 2 cases of VSD, a case of dextrocardia, a case of HLHS, a case of tetralogy of Fallot and a case of cardiomegaly with pericardial effusion) (Table 2) .

Two cases of those with cardiac abnormalities were found in the high-risk subgroup, constituting 5.5\% of the high-risk subgroup; (a case of dextrocardia and other of cardiomegaly with pericardial effusion). The other 4 abnormal cases were of low risk, constituting $3.5 \%$ of the low-risk subgroup (Table 3).

- The four-chamber view was the main view for diagnosis of 5 fetuses with cardiac anomalies; two cases with ventricular septal defect, one with dextrocardia, one with hypoplastic left heart syndrome, and one with cardiomegaly and pericardial effusion while extended echocardiographic evaluation of ventriculo-arterial connections; namely the LVOT view was the main view for detection and diagnosis of other cardiac anomaly in an additional fetus; outlet ventricular septal defect associated with large overriding aortic root, which is pathognomonic of tetralogy of Fallot.

- Of the examined 150 fetuses, 3 cases were found to have a left ventricular echogenic focus; however they were not considered as positive cases being variation of normal.

- Color Doppler was the mainstay for diagnosis of a case of VSD, which was not obviously seen on the 2D-gray scale US; on application of color flow Doppler, we could detect color flow across the septum. Color Doppler also helped differentiating cases with true inlet VSDs from cases misinterpreted as VSDs in the 2D-gray scale apical four-chamber view where the septum was viewed with the beam parallel to its long axis; the normal transition from muscular to membranous septum resulted in signal dropout.

- In a case of tetralogy of Fallot, color Doppler showed the aortic root receiving blood from both ventricles and another case of HLHS, there was no flow across 
the mitral valve to the hypoplastic left ventricle.

Table (1): Stratification of high-risk cases

\begin{tabular}{lc}
\hline & $\mathbf{n}(\%)$ \\
\hline Age $>\mathbf{3 5}$ & $9(6)$ \\
Diabetes mellitus & $4(2.7)$ \\
TORCH & $2(1.3)$ \\
Positive consanguinity & $15(10)$ \\
Past history of CHD & $2(1.3)$ \\
Assisted conception & $3(2)$ \\
Mono-chorionic twin & $1(0.6)$ \\
\hline
\end{tabular}

Table (2): Cardiac anomalies detected among the study population by prenatal echocardiographic examination

\begin{tabular}{|c|c|c|}
\hline \multicolumn{2}{|l|}{ Anomaly: } & n $(\%)$ \\
\hline \multicolumn{2}{|l|}{ VSD } & $2(1.3 \%)$ \\
\hline \multicolumn{2}{|l|}{ Dextrocardia } & $1(0.6 \%)$ \\
\hline \multicolumn{2}{|c|}{ Cardiomegaly with pericardial effusion } & $1(0.6 \%)$ \\
\hline \multicolumn{2}{|l|}{ Tetralogy of Fallot } & $1(0.6 \%)$ \\
\hline \multicolumn{2}{|l|}{ HLHS } & $1(0.6 \%)$ \\
\hline \multicolumn{2}{|l|}{ No anomaly } & $144(96 \%)$ \\
\hline \multicolumn{3}{|c|}{$\begin{array}{l}\text { VSD }=\text { Ventricular septal defect } \\
\text { HLHS }=\text { Hypoplastic left heart syndrome }\end{array}$} \\
\hline \multicolumn{3}{|c|}{$\begin{array}{l}\text { Table (3): Distribution of positive cases among the } \\
\text { high- and low-risk subgroups }\end{array}$} \\
\hline Group & $\begin{array}{l}\text { Number of } \\
\text { cases } \\
\text { investigated } \\
\quad(n=150)\end{array}$ & $\begin{array}{l}\text { Number of } \\
\text { cases } \\
\text { diagnosed } \\
(n=6)\end{array}$ \\
\hline High-risk group: & $36(24 \%)$ & $\underline{2(5.5 \%)}$ \\
\hline $\begin{array}{l}\text { - Maternal age older than } \\
35 \text { years }\end{array}$ & 9 & $\mathbf{0}$ \\
\hline -Positive consanguinity & 15 & $\mathbf{0}$ \\
\hline $\begin{array}{l}\text { - Co-existing maternal } \\
\text { disease. }\end{array}$ & 6 & 1 \\
\hline $\begin{array}{l}\text { - Previous baby with } \\
\text { CHD. }\end{array}$ & 2 & $\mathbf{0}$ \\
\hline - Assisted conception & 3 & $\mathbf{0}$ \\
\hline -Mono-chorionic twin & 1 & 1 \\
\hline
\end{tabular}

\section{Discussion}

CHDs are the most common major structural fetal abnormalities, affecting approximately eight per 1000 live births in Europe. Half of the CHDs are major cardiac defects. ${ }^{(14)}$

Prenatal diagnosis of CHDs decreases mortality and morbidity by smooth transition between pre- and post-natal states by providing the appropriate medical care immediately after birth. ${ }^{(15)}$

Although many risk factors for CHD have been identified, more than $90 \%$ of cases occur in low-risk patients. ${ }^{(16)}$ Therefore, the guidelines of several ultrasound societies recommend second-trimester fetal screening for CHD for the entire population of pregnant women. ${ }^{(17)}$

Fetal cardiac screening assessment via ultrasound commonly includes assessment of the four-chamber view and ventricular outflow tracts although it may be more extensive depending on the examiner. ${ }^{(1)}$

Detection of CHD may lead to the detection of other structural fetal anomalies or karyotypic abnormalities. Commoner examples of this include the detection of trisomy 21 following diagnosis of a complete atrioventricular septal defect (AVSD) or a 22q11 deletion following detection of tetralogy of Fallot. ${ }^{(18)}$ 
Our study aimed to assess the role of a detailed fetal echocardiography in prenatal detection of CHD in an unselected population of pregnant women.

CHD was detected by a detailed fetal echocardiography in 6 of the 150 fetuses included in the study with overall incidence $4 \%$, which is higher than that found in general population (8 per 1,000 live births); this discrepancy between pediatric and prenatal series may be attributed to the high lethality rate of some malformations in the perinatal period.

In a previous similar study by Özkutlu et al. (19) they also detected a much higher prevalence of CHD to be as (10.9\%) at 1395 fetuses; their study population included lowand high-risk pregnant women.

Sixty six point six percent $(66.6 \%)$ of fetuses diagnosed with CHD (4 cases) were found in the low-risk group, while only 2 cases (33.3\% of fetuses with CHD) were in the high-risk group. Our results in low-risk group agreed with the results of a previous study by Özkutlu et al. in which $56.6 \%$ of the fetuses with CHD were in the low-risk group. ${ }^{(19)}$

Regarding the prevalence of the detectable CHD in each subgroup separately; we found that the intra-uterine heart disease prevalence was $5.5 \%$ in the high-risk group and $3.5 \%$ in the low-risk group $(\mathrm{P}=0.4)$, so there was no statistically significant difference in the incidence of CHD between pregnancies associated with high risk factors compared to low risk pregnancies. Our results in the high- and low-risk subgroups were matching with the study of Nayak et al. (4) that was also conducted on an unselected group of population; it reported that high proportion of prenatally detectable cases of CHD (24 of 26 cases) occurred in the low-risk group with incidence of $2.2 \%$ compared to $1.6 \%$ in the high-risk group. The difference in the incidence of CHD between the two groups was not statistically significant $(\mathrm{P}=0.76)$.

Two other studies yielded a statistically significant increased risk of congenital cardiac defects in the low-risk subgroups $(\mathrm{P}<0.001)$; one by Perri et al., ${ }^{(20)}$ they found that most of cardiac anomalies (41 of $46 ; 89 \%$ ) were in the low risk group. The second by Özkutlu et al. ${ }^{(19)}$ which stated that CHD prevalence was $19 \%$ in the low-risk group and only $7 \%$ in the high-risk group, and as most of CHDs in their study were determined in pregnancies of low risk (86 out of 152 cases); they assigned the mission 
of prenatal detection of CHD to gynecologists and obstetricians.

Our results were fairly matching with results of Özbarlas et al. ${ }^{(21)}$ regarding the overall incidence of the prenatally detected CHD by fetal echo-cardiography and the most frequently observed cardiac anomaly; they showed that the incidence of structural cardiac anomalies was $5.6 \%$ in all pregnant women; $7.8 \%$ in the high-risk group and $2.7 \%$ in the low risk group, (compared to $5 \%$ and $3.6 \%$ in the high- and low-risk groups in our study respectively).

In a study by Hui et al. (22), they used the same five transverse planes used in our study according to Yagel et al. (2001)'s description $^{(23)}$ and detected CHD rate of $4.68 \%$. However, the data in their study were drawn from a group with a high risk for congenital heart disease. This incidence was concordant with the incidence of CHD detected in our high-risk group (5\%).

Our results in the high-risk group did not agree with the results of a study conducted by Rocha et al. ${ }^{(24)}$ who showed that the incidence of CHD in fetuses was $9.9 \%$, their study population were pregnant women who were referred for suspicion or with some risk of $\mathrm{CHD}$.
The most common encountered CHD in our study was isolated VSD; 33\%. These results were matching well with that of Özbarlas et al. ${ }^{(21)}$ which found that isolated VSD was the most frequently detected cardiac anomaly representing $36.2 \%$ of all cardiac anomalies.

Özkutlu et al. ${ }^{(19)}$ stated also that the most frequently structural CHDs detected in their study was isolated VSD with incidence of $16.7 \%$. Perri et al. ${ }^{(20)}$ reported a higher percentage of tetralogy of Fallot (19.6\%) than VSD (17.4\%); this did not agree with our results.

In our study, we detected 3 cases with left ventricular echogenic focus and none of them had any cardiac abnormality, this agreed with a study by Özkutlu et al., (19) they detected 17 cases with ventricular echogenic focus (15 LV, $2 \mathrm{RV}$ ) in otherwise normal heart and Perri et al. ${ }^{(20)}$ who also observed that only one case of 238 fetuses with intra-cardiac echogenic focus, have a cardiac anomaly (VSD).

Our study showed that $83.3 \%$ of the detectable cardiac abnormalities were diagnosed based on the four-chamber view alone; however, a case of over-riding aortic root over a VSD that is pathognomonic for tetralogy of Fallot could not be diagnosed in 
the four-chamber view alone. Such a case would have been missed if we had not incorporated the outflow tracts in our examination protocol.

Our results agreed with a study conducted by Vergani et al. ${ }^{(25)}$ which reported detection rate $81 \%$ by the four-chamber view, their study population were of mixed risk. Copel et al. (26) also examined 1022 fetuses and detected 74 structurally abnormal hearts; 71 of these $(96 \%)$ were diagnosed in the fourchamber view.

A multi-center study conducted by Ogge et al. ${ }^{(27)}$ showed that the addition of the outflow-tract view in the prenatal ultrasound screening for $\mathrm{CHD}$ had improved the performance and allowed the detection of malformations not recognizable with the four-chamber view. This was consistent with what we have found that addition of the outflow tract views had an impact on the detection of anomalies not recognizable on the four-chamber view alone.

In addition, a meta-analysis was conducted by li et al. ${ }^{(28)}$ on the performance of different scan protocols of fetal echocardiography, concluded that at least 3 sections view (4chamber view, outflow tracts view and 3vessel tracheal view) should be included in any scan protocol of fetal echocardiographic examination to be a reliable method for prenatal detection of congenital heart disease. This agreed with the examination protocol we followed in our study.

\section{Limitations of the study}

Our results would have been more representative if we had a larger study population, so we could have caught more number of cases with abnormal findings especially those involving the outflow tracts.

\section{Recommendations}

The value of fetal echocardiography as a screening method for prenatal detection of congenital heart disease would be more obvious in a larger population; we propose further studies to cover a higher percentage of population.

As most of cardiac anomalies observed in our study were detected in the low-risk group and given the morbidity and mortality associated with CHD, we recommend that at least both of the 4-chamber view and outflow tract views to be standardized in any fetal anomaly scanning for every pregnant woman regardless the presence of risk factors. This requires more efforts to be made for increasing awareness about fetal echocardiography among both radiologists and obstetricians.

\section{Conclusion}

Fetal echocardiography may be a valuable tool in prenatal detection of CHDs even in 
low-risk population and the four-chamber view of the fetal heart was the main view for detection of most of cardiac abnormalities.

\section{References}

1. Vavolizza, R. D., Dar, P. E., Suskin, B., Moore, R. M., \& Stern, K. W. Clinical yield of fetal echocardiography for suboptimal cardiac visualization on obstetric ultrasound. Congenital heart disease. 2018; 13(3): 407-412.

2. Lambert, T. E., Kuller, J., Small, M., Rhee, E., \& Barker, P. Abnormalities of fetal situs: an overview and literature review. Obstetrical \& gynecological survey. 2016; 71(1): 33-38.

3. Hoffman, J. I., \& Kaplan, S. The incidence of congenital heart disease. Journal of the American college of cardiology. 2002; 39(12): 1890-1900.

4. Nayak, K., Naveen Chandra, G. S., Shetty, R., \& Narayan, P. K. Evaluation of fetal echocardiography as a routine antenatal screening tool for detection of congenital heart disease. Cardiovascular diagnosis and therapy. 2016; 6(1): 44.

5. Allan, L. D. Fetal echo-cardiography. Surgery for congenital heart defects. 2th. London. 1994; 8586.

6. Sharland, G. Fetal cardiology. In Seminars in neonatology. (2001, February); (Vol. 6, No. 1, pp. 315). Elsevier.

7. Allan, L. D. A Practical approach to fetal heart scanning. In Seminars in perinatology. (2000, October); (Vol. 24, No. 5, pp. 324-330). Elsevier.

8. Allan LD, Crawford DC, Chita SK, Tynan MJ. Prenatal screening for congenital heart disease. $\mathrm{Br}$ Med J 1986; 292: 1717-1719.

9. Fermont L, De Geeter B, Aubry MC, Kachener

J, Sidi D. A close collaboration between obstetricians and cardiologists allows antenatal detection of severe cardiac malformations by 2D echocardiography (abstract). Proceedings of the Second World Congress of Paediatric Cardiology. New

York 1986; 10.

10. Sharland GK, Allan LD. Screening for congenital heart disease prenatally. Results of a 221 year study in the South East Thames Region. $\mathrm{Br} J$ Obstet Gynaecol 1992; 99: 220-225.

11. Donofrio MT, Moon-Grady AJ, Hornberger LK, Copel JA, Sklansky MS, Abuhamad A, et al; American Heart Association Adults With Congenital Heart Disease Joint Committee of the Council on Cardiovascular Disease in the Young and Council on Clinical Cardiology, Council on Cardiovascular Surgery and Anesthesia, and Council on Cardiovascular and Stroke Nursing. Diagnosis and treatment of fetal cardiac disease a scientific statement from the American Heart Association. Circulation. 2014; 129(21):2183-242.

12. Eggebø, T. M., \& Heien, C. An easy way to determine fetal heart laterality. Acta obstetricia et gynecologica Scandinavica, 2010; 89(1): 159-160.

13. Carvalho, J. S., Allan, L. D., Chaoui, R., Copel, J. A., DeVore, G. R., Hecher, K., ... \& Yagel, S. ISUOG Practice Guidelines (updated): sonographic screening examination of the fetal heart. Ultrasound in Obstetrics \& Gynecology, 2013; 41(3): 348-359.

14. Fernández, S. G., Ramirez, J. A., Chouza, M. T. O., Alonso, B. R. V., \& Coto, Á. P. L. Early fetal ultrasound screening for major congenital heart defects without Doppler. European Journal of Obstetrics \& Gynecology and Reproductive Biology, 2019; 233: 93-97.

15. Satomi, G. Guidelines for fetal echocardiography. Pediatrics International 2015; 57(1): 1-21.

16. Bishop, K. C., Kuller, J. A., Boyd, B. K., Rhee, E. H., Miller, S., \& Barker, P. Ultrasound examination of the fetal heart. Obstetrical \& gynecological survey, 2017; 72(1): 54-61. 
17. Sarkola, T., Ojala, T. H., Ulander, V. M., Jaeggi, E., \& Pitkänen, O. M. Screening for congenital heart defects by transabdominal ultrasound-role of early gestational screening and importance of operator training. Acta obstetricia et gynecologica Scandinavica, 2015; 94(3): 231-235.

18. Simpson, J., Zidere, V., \& Miller, O. I. (Eds.). Fetal Cardiology: A Practical Approach to Diagnosis and Management. Springer.2018

19. Özkutlu, S., Akça, T., Kafali, G., \& Beksaç, S. The results of fetal echocardiography in a tertiary center and comparison of low-and high-risk pregnancies for fetal congenital heart defects, 2010; 10(3):263

20. Perri, T., Cohen-Sacher, B., Hod, M., Berant, M., Meizner, I., \& Bar, J. Risk factors for cardiac malformations detected by fetal echocardiography in a tertiary center. The Journal of Maternal-Fetal \& Neonatal Medicine, 2005; 17(2): 123-128.

21. Özbarlas, N., Erdem, S., Küçükosmanoğlu, O., Seydaoğlu, G., Demir, C., Evrüke, C., \& Özgünen, F. T. Prevalence and distribution of structural heart diseases in high and low risk pregnancies. Anatolian Journal of Cardiology/Anadolu Kardiyoloji Dergisi, 2011; 11(2).

22. Hui, L., Jun, W., Ying, M., \& Tao, S. Prenatal diagnosis of congenital fetal heart abnormalities and clinical analysis. Journal of Zhejiang University Science B, 2005; 6(9): 903-906.

23. Yagel, S., Cohen, S.M., Achiron, R. Examination of the fetal heart by five short axis views: A proposed screening method for comprehensive cardiac evaluation.Ultrasound Obstet. Gynecol., 2001; 17(5): 367-369.

24. Rocha, L. A., Araujo Júnior, E., Rolo, L. C., Barros, F. S. B., da Silva, K. P., Leslie, A. T. F. S., ... \& Moron, A. F. Prenatal detection of congenital heart diseases: one-year survey performing a screening protocol in a single reference center in Brazil. Cardiology research and practice, 2014.

25. Vergani, P., Mariani, S., Ghidini, A., Schiavina, R., Cavallone, M., Locatelli, A., ... \& Cerruti, P. Screening for congenital heart disease with the fourchamber view of the fetal heart. American journal of obstetrics and gynecology, 1992 ;167(4): 10001003.

26. Copel, J. A., Pilu, G., Green, J., Hobbins, J. C., \& Kleinman, C. S. Fetal echocardiographic screening for congenital heart disease: the importance of the four-chamber view. American journal of obstetrics and gynecology, 1987; 157(3): 648-655.

27. Ogge, G., Gaglioti, P., Maccanti, S., Faggiano, F., \& Todros, T. Prenatal screening for congenital heart disease with four-chamber and outflow-tract views: a multicenter study. Ultrasound in Obstetrics and Gynecology: The Official Journal of the International Society of Ultrasound in Obstetrics and Gynecology 2006; 28(6): 779-784.

28. Li, Y., Hua, Y., Fang, J., Wang, C., Qiao, L., Wan, C., ... \& Zhou, K. Performance of different scan protocols of fetal echocardiography in the diagnosis of fetal congenital heart disease: a systematic review and meta-analysis. PLoS One, 2013; 8(6): e65484.

To cite this article: Hesham El Sheikh, Ahemd E. Shalaan, Esraa A. Abdelazeem. Fetal Echocardiography as a Screening Method for Detection of Congenital Heart Disease. BMFJ 2021;38(Radiology):11-21. DOI: 10.21608/bmfj.2020.16104.1039 